\title{
PSYCHOLOGY RESEARCH SEMINAR ABSTRACTS
}

The following are abstracts from the research seminar series presented March - June 1987 in the Department of Psychology and Philosophy at the University of Papua New Guinea. Further details of the contents of individual seminars may be obtained from the authors.

\section{Drugs at Work: The Effects of Betel Nut Chewing on Selected Psychophysiological Variables}

\author{
Thomas Wyatt \\ Department of Psychology and Philosophy \\ University of Papua New Guinea*
}

Thirty-one male earth-moving operators, with an average age of 30 years, volunteered to participate in a lab study of the impact of chewing various quantities of betel nut upon visual-choice reaction time, digit span, eye-hand co-ordination, pulse rate, and blood pressure. Because of the paucity of published research and conflicting statements about the nature of the effects of betel nut chewing, all hypotheses were subjected to two-tailed tests. Using a one-way analysis of variance repeated-measures design, the results indicated no statistical effect of betel nut chewing upon visual-choice reaction time, digit span, eye-hand co-ordination (as measured by the pursuit rotor task), systolic or diastolic blood pressure. Results did show a statistically significant impact on pulse rate in a positive direction. The discussion focuses on the direct psychophysiological effects of betel nut chewing upon the person. Given the fact that the results indicate no significant decrement in the workrelated dependent variables, a discussion ensues on the possible use of betel nut at the work place and particularly as an antidote for driver fatigue.

*Now at National University of Singapore.

\section{An Observational Study of the Psychiatric Ward at Port Moresby General Hospital \\ Albert Mellam \\ Department of Psychology \& Philosophy \\ University of Papua New Guinea}

The prime purpose of this clinical research was to test the goodness of fit of any one clinical case to the existing theories and nosologies of severe depression. The research was conducted on patients examined and treated in the psychiatric clinic at the Port Moresby General Hospital. It involved observations, interviews, history-takings, and examinations of patients, and attempts at diagnoses. It was not possible to obtain a severe depressive case 
and it was therefore not possible to determine whether an individual case would fit with any one of the existing theories and classifications of severe depression. Multiaxial syndromes of mental disorders, out-dated diagnostic procedures, and problems in ward and patient management may have hindered the attempt to obtain a diagnosed depressed case for the purpose of study. It is these observations that constitute the discussion in this seminar.

\title{
Brain Lateralization and Selected Cognitive Functions in Second- Language Speakers
}

\author{
Dianne Wuillemin \\ Department of Psychology \& Philosophy \\ University of Papua New Guinea
}

In a series of experiments, Papua New Guinean multilingual students were tested for language laterality (in English and Tok Pisin) and on a variety of proficiency, cognitive and perceptual tasks. Acquisition age effects were observed for every measure taken except one (a concurrent finger-tapping and speaking task) such that:

involvement of the right hemisphere in language processing increased with increasing acquisition age of the language for both English and Tok Pisin.

older acquirers performed significantly worse than younger acquirers on proficiency tests (vocabulary, spelling, grammar, and comprehension). This could not be accounted for by length of time that the language had been known.

older acquirers remembered fewer items on both iconic and short term memory tests and displayed an unusual serial position effect, lacking in recency, when compared with younger acquirers.

a significant 'reverse' effect (whereby pictures interfered with word naming) was found for the older acquirers, but not for the younger acquirers, on a Stroop-type, picture-word interference task.

older acquirers were significantly slower at naming both visually and auditorally presented words, and at making lexical decisions, and were less accurate at recognizing briefly presented words.

All of the above effects were tested only in English unless otherwise stated. No relationship was found between laterality for English and performance on any of the other tasks. Laterality for visuospatial processing was also determined and was compared for subjects having different second-language (regardless of which one) acquisition ages. Late second-language acquirers showed the usual right hemisphere advantage for visuospatial processing, 
whereas early acquirers displayed no hemispheric advantage. The results of the visuospatial experiment taken together with the wide range of deficits reported in language-based tasks above, highlight the importance of early second-language learning, and, in a country which relies so heavily on English in its education system, emphasizes the need for early English instruction in PNG schools.

\title{
Assessment and Description of Mental Retardation in Papua New Guinean Schools
}

\author{
Jean Eparo \\ Department of Psychology \& Philosophy \\ University of Papua New Guinea
}

\begin{abstract}
Assessment and description of mental retardation is based on results of tests that are given to individuals. One of the tests used widely in Western countries is the Wechsler Intelligence Scale for Children (WISC), which is constructed for school children whose ages range from 6 to 16 years. WISC has two major parts: Performance and Verbal, and each has 6 sub-tests. The reliability and validity of WISC are very high. This test could be used in PNG schools to assess mental retardation. However, results of an Australian version of WISC

given to two very bright children in a community school in Gerehu showed that they were mentally retarded indicating that the WISC constructed for Western children is not valid for PNG children. What is needed is a restandardised WISC based on materials used in PNG culture for PNG children.
\end{abstract}

\section{The Influence of Age, Anxiety, and Time of Day on the Critical Flicker Fusion Threshold}

\author{
Lynn Frewer \\ Department of Psychology and Philosophy \\ University of Papua New Guinea
}

The Critical Flicker Fusion Threshold (CFFT) is extensively used in psychopharmacological research as a measure of change induced by the administration of psychotropic agents, insomuch as the threshold tends to be increased following the administration of CNS stimulants, and decreased following the administration of CNS depressants, which led to the notion that the CFFT acts as an indicator of CNS activation or arousal. Discrepancies in previous review papers have been explained with reference to the adoption of inappropriate experimental methodologies in research, or problems which arise from making inter-study comparisons where data have been collected using different methods of CFFT assessmeit betveen trials. A meta-analysis 
of archival data, where methodologies were constant across trials and incorporated the use of appropriate controls was made. The influence of a range of psychotropic drugs on the CFFT (including acute dose, repeated dose and withdrawal data) was examined. It was found that CFFT was reliable, in the sense that a specific substance tended to produce a unidirectional change in the CFFT, and that the magnitude of this change was consistent with the initial dose level and exhibited time-course effects appropriate to a given drug.

It was not apparent how this related to changes in CNS activation, however. The influence of other external variables such as anxiety level and age, which are of psychopharmacological relevance, were examined. The literature seemed to indicate that both high levels of anxiety, and increased chronological age result in a decrease in CFFT. In the instance of increased anxiety levels there was sufficient evidence, in the form of previously published experimentation, to indicate that higher levels of anxiety resulted in lower CFFT values. Previous investigations of the relationship between age and CFFT were less well controlled, and so an archival analysis of data arising in studies where relevant factors (such as the use of psychotropic substances in the subject population) had been controlled was performed. This study indicated that a linear relationship between age and CFFT existed, where CFFT declined with increased chronological age. A further study indicated that the differences in CFFT resulting between high and low anxiety groups, and young and old subject populations, were reliable, and occurred at all times during the diurnal period. Evidence from psychopharmacological research, and the use of the psychophysical method of limits, would seem to indicate that changes in CFFT were central in origin. A diurnal decline in CFFT was also observed. Taken together, it was suggested that these results indicate that the traditional "arousal" model of CFFT was inappropriate, and that it might be useful to think of CFFT as being dependent on the efficiency of early, precatagorical stages in the visual information processing system. 